УдК

\title{
СЖИМАЮЩИЙ КАНАЛЬНЫЙ ЭКВАЛАЙЗЕР ДЛЯ СЕТЕЙ МНОЖЕСТВЕННОГО ДОСТУПА ТН-UWВ С МНОГОЛУЧЕВЫМ РАСПРОСТРАНЕНИЕМ ПРИ НАЛИЧИИ МНОГОПОЛЬЗОВАТЕЛЬСКОЙ ИНТЕРФЕРЕНЦИИ
}

\author{
НУРЕДДИН Б. БЕНОТМАН, СИДАХМЕД ЭЛАХМАР
}

Университет Джилали Лиабеса, Алжир, Сиди-Бель-Аббес

\begin{abstract}
Аннотация. В статье представлен новый сжимающий канальный эквалайзер CSE (channel shortening equalizer) для сверхширокополосных (СШП) систем множественного доступа с переключением временных каналов с применением модуляции положения импульса РPM (pulse position modulation). Поскольку СШП каналы имеют очень длинную импульсную характеристику по сравнению с шириной узкого импульса, то применение CSE способствует уменьшению числа корреляторов приемника. В СШП системах благодаря тому, что полученный импульс подобен импульсной характеристике канала, предложенный алгоритм максимизирует отношение сжатого сигнала к межсимвольной и многопользовательской интерференции. Наличие предложенного CSE перед корреляционным приемником уменьшает сложность архитектуры приемника за счет уменьшения количества эффективных канальных отводов. В дальнейшем предложенный метод использован для получения общего выражения частоты появления ошибочных битов BER (bit error rate) при наличии межсимвольной и многопользовательской интерференции. Приведены результаты компьютерного моделирования производительности предложенного метода в сравнении с методом MSSNR CSE, а также с нижней границей, известной как метод all-rake, и методами partial-rake, и selective-rake по таким параметрам, как действующее значение временного окна rake-приемника и BER.
\end{abstract}

Ключевые слова: сверхширокополосный; СШП; UWB; сжимающий эквалайзер; rake приемник

\section{1. ВВЕДЕНИЕ}

Импульсная сверхширокополосная (СШП) радиосеть с переключением временных каналов ТН (time-hopping) - это развивающаяся технология сетей высокоскоростной передачи данных для ближней многопользовательской коммуникации [1-4]. Для СШП систем множественного доступа с ТН возможность получения высокой скорости передачи данных с относительно небольшой сложностью и низким энергопотреблением является ключевым требованием.

Благодаря значительной задержке распространения, на производительность СШП системы с переключением временных каналов
TH-UWB (TH ultra-wideband) влияют многопользовательская интерференция MUI (multiuser interference) и межкадровая интерференция IFI (inter-frame interference), которая также включает межсимвольную интерференцию ISI (inter-symbol interference) [5, 6].

Rake-приемник (приемник разнесенных сигналов) обычно состоит из множества корреляторов для увеличения принятого отношения сигнал/шум и ослабления затухания канала [6-9]. Однако наибольшее внимание при разработке СШП rake-приемника уделяется количеству комбинируемых путей, тогда как возрастание сложности происходит с увеличением числа под-приемников (finger). 


\section{БИБЛИОГРАФИЧЕСКИЙ СПИСОК}

1. Yang, L.; Giannakis, G. B. Ultra wide band communication: An idea whose time has come. IEEE Signal Proc. Mag., v.21, n.6, pp. 26-54, Nov. 2004. DOI: 10.1109/MSP.2004.1359140.

2. Hazra, R.; Tyagi, A. Cooperative impulse radio ultra-wideband communication using coherent and non-coherent detectors: A review. Wireless Pers. Commun. Vol. 77, n. 1, pp. 719-748, 2014. DOI: $10.1007 /$ s11277-013-1533-x.

3. Дубровка, Ф. Ф.; Третьяков, В. А. Анализ спектральной эффективности цифровых сигналов для сверхширокополосных радиосистем диапазона частот 3,1-10,6 ГГц. Известия вузов. Радиоэлектроника, Т. 54, № 9, C. 3-11, 2011. URI: http://radio.kpi.ua/article/ view/S0021347011090019.

4. Win, M. Z.; Scholtz, R. A. Ultra-wide bandwidth time-hopping spread-spectrum impulse radio for wireless multiple-access communications. IEEE Trans. Commun., v.48, n.4, pp. 679-691, Apr. 2000. DOI: $10.1109 / 26.8$ $\underline{43135}$.

5. Fang, L.; Zailu, H. Low complexity multi-user detectors of TH-PPM UWB system. Proc. of Int. Conf. on Wireless Commun., Netw. and Mob. Comp., 26 Sept. 2005, Wuhan, China. IEEE, 2005, pp. 320-323. DOI: 10.1109/ WCNM.2005.1544041.

6. Zhang, L.; Yang, C. Performance analysis of channel shortening of RAKE receiver in ultra-wideband systems. Proc. of 64th Veh. Tech. Conf., VTC, 25-28 Sept. 2006, Montreal, Que., Canada. IEEE, 2006, pp. 1-5. DOI: 10.1109/VTCF.2006.166.
7. Zhang, L.; Yang, C. The equalization performance of RAKE receiver in UWB system. Proc. of 7th Int. Conf. on Sig. Process., ICSP, 31 Aug.-4 Sept. 2004, Beijing, China. IEEE, 2004, pp. 1723-1726. DOI: 10.1109/ICOSP. 2004.1441667.

8. Husain, S. I.; Yuan, J.; Zhang, J. Rake performance after channel shortening by decay factor optimization in UWB channels. Proc. Of 66th IEEE Veh. Tech. Conf., VTC, 30 Sept.-3 Oct. 2007, Baltimore, USA. IEEE, 2007, pp. 1204-1207. DOI: 10.1109/VETECF. 2007.259 .

9. Cassioli, D.; Win, M. Z.; Vatalaro, F.; Molisch, A. F. Performance of low-complexity RAKE reception in a realistic UWB channel. Proc. of IEEE Int. Conf. on Commun., ICC, 28 Apr.-2 May 2002, New York, USA. IEEE, 2002, pp. 763-767. DOI: 10.1109/ICC.2002.99 6958.

10. Martin, R. K.; Vanbleu, K.; Ding, M.; Ysebaert, G.; Milosevic, M.; Evans, B. L.; Moonen, M.; Johnson, C. $\mathrm{R}$. Unification and evaluation of equalization structures and design algorithms for discrete multitone modulation systems. IEEE Trans. Signal Process., Vol. 53, No. 10, pp. 3880-3894, 2005. DOI: 10.1109/TSP.2005.855432.

11. Melsa, P. J. W.; Younce, R. C.; Rohrs, C. E. Impulse response shortening for discrete multitone transceivers. IEEE Trans. Commun., Vol. 44, n. 12, pp. 1662-1672, 1996. DOI: $10.1109 / 26.545896$.

12. Ragoubi, K.; Hélard, M.; Crussière, M. Low complexity channel shortening technique applied to MB-OFDM UWB systems. Proc. of 4th Int. Conf. on Signal Process. and Commun. Systems, ICSPCS, 13-15 Dec. 2010, Gold Coast, QLD, Australia. IEEE, 2010, pp. 1-6. DOI: 10.1109/ICSPCS.2010.5709684.

13. Chopra, A.; Evans, B. L. Design of sparse filters for channel shortening. Proc. of Int. Conf. on Acoustics Speech and Signal Process., ICASSP, 14-19 Mar. 2010, Dallas, TX, USA. IEEE, 2010. DOI: 10.1109/ICASSP. 2010.5495507.

14. Avinash, M.; Hari, K. V. S. "Low complexity adaptation for SISO channel shortening equalizers. $A E U$ - Int. J. Electron. Commun., vol. 66, no. 8, pp. 600-604, 2012. DOI: 10.1016/j.aeue.2012.03.011.

15. Berriah, Boubakeur; Bouziani, Merahi; Elahmar, S. A. New blind, adaptive channel shortening TEQ for multicarrier modulation systems. IET Commun., Vol. 8, No. 2, pp. 210-216, 2014. DOI: 10.1049/iet-com.2013. 0147.

16. Wu, W. R.; Lee, C. F.; Chen, Y. F. Time-domain equalisation for discrete multi-tone transceivers: new results and performance analysis. IET Commun., Vol. 3, No. 7, pp. 1186-1200, 2009. DOI: 10.1049/iet-com.2008. $\underline{0133}$.

17. Husain, S. I.; Choi, J. Single correlator based UWB receiver implementation through channel shortening equalizer. Proc. of Asia-Pacific Conf. on Commun., 5 Oct. 2005, Perth, WA, Australia. IEEE, 2005, pp. 610-614. DOI: 10.1109/APCC.2005.1554134. 
18. Husain, S. I.; Choi, J. Blind adaptive channel shortening by unconstrained optimization for simplified UWB receiver design. Proc. of 3rd Int. Symp. on Wireless Commun. Systems, ISWCS, 6-8 Sept. 2006, Valencia, Spain. IEEE, 2006, pp. 443-446. DOI: 10.1109/ISWCS. 2006.4362336.

19. Husain, S. I.; Yuan, J.; Zhang, J. Modified channel shortening receiver based on MSSNR algorithm for UWB channels. Electron. Lett., Vol 43, No. 9, pp. 535-537, Apr. 2007. DOI: 10.1049/el:20070584.

20. Husain, S. I.; Yuan, J.; Zhang, J.; Martin, R. K. Time domain equalizer design using bit error rate minimization for UWB systems. EURASIP J. Wireless Commun. Networking, vol. 2009, Article ID 786291, 11 pages, 2009. DOI: $\underline{10.1155 / 2009 / 786291}$.
21. Foerster, J. R.; et al. Channel modelling sub-committee report final. Tech. Rep. IEEE P802.15-02/490r1 SG3a, Working Group for Wireless Personal Area Networks, Monterey, Calif, USA, Feb. 2003.

22. Feng, D.; Ghauri, S.; Zhu, Q. Application of the MUI model based on packets collision (PC) in UWB ad-hoc network. Proc. of Int. Conf. on Networking, Sensing and Control, 26-29 Mar. 2009, Okayama, Japan. IEEE, 2009. DOI: 10.1109/ICNSC.2009.4919337.

23. Durisi, G.; Benedetto, S. A. Performance evaluation of TH-PPM UWB systems in the presence of multiuser interference. IEEE Commun. Lett., Vol. 7, No. 5, pp. 224-226, 2003. DOI: 10.1109/LCOMM.2003.812171. 